\title{
Nasal epithelium: new insights and differences of the cytokine profile between normal subjects and subjects with allergic rhinitis*
}

\author{
S. Stamataki',2, N.G. Papadopoulos33, J. Lakoumentas33, A. Georgountzou3, $\quad$ Rhinology Online, vol 4: 223-232, 2021 \\ P. Maggina3 ${ }^{3}$ P. Xepapadaki ${ }^{3}$, E. Andreakos ${ }^{4}$, E. Prokopakis², E. Legaki ${ }^{3}$, S. Taka3 http://doi.org/10.4193/RHINoL/21.047
}

' ENT Department, Childrens' Hospital "Agia Sofia”, Athens, Greece

2 ENT- Head and Neck Surgery Department, University of Crete School of Medicine, Heraklion, Crete, Greece

${ }^{3}$ Allergy and Clinical Immunology Unit, Second Department of Pediatrics, National and Kapodistrian University of Athens,

Athens, Greece

${ }^{4}$ Department of Immunology, Center for Translational and Clinical Research, Biomedical Research Foundation, Academy of Athens, Athens, Greece

\begin{abstract}
Background: The role of the nasal epithelium in the induction of a proper cytokine response in normal subjects and subjects with allergic rhinitis is still not completely elucidated.

Methodology: We aimed to compare nasal epithelial immune responses in allergic rhinitis patients of different ages compared to healthy volunteers. Primary nasal epithelial cells from 47 subjects ( 33 normal and 17 with allergic rhinitis) were collected and cultured. Their unstimulated supernatants were analysed for 21 cytokines and chemokines. Statistical analysis was performed with the R statistical software and the RStudio interface.
\end{abstract}

Results: Differences of the spontaneous release of epithelial cytokines and chemokines were noticed between the two study groups. The levels of GMCSF, MIP1A, MIP1B, IL28A, TNFA, CCL5 were significantly lower in the allergic rhinitis group compared to healthy volunteers' group, independent of age. Most differences were noticed in the younger allergic rhinitis group (0-12 years old).

Conclusions: Despite the cross-sectional nature of the study and the limited number of subjects, allergic rhinitis appears to be associated with dysfunction of cytokine and chemokine spontaneous release from nasal epithelial cells which may represent an abnormal innate immunity maturation pattern.

Key words: allergic rhinitis, chemokines, cytokines, innate immunity, nasal mucosa

\section{Introduction}

Despite the dramatic increase of allergic rhinitis (AR), affecting $20 \%-30 \%$ of the global population ${ }^{(1)}$, and the intense study of the role of allergy and innate immunity underpinning its pathophysiology, there are still critical knowledge gaps ${ }^{(2)}$. Several studies, mostly on allergic asthma, have explored the significance of the airway epithelium on the induction of an appropriate immune response towards potentially harmful inhaled substances and microbial pathogens or driving the downstream innate responses. However, there are limited studies focusing on the nasal epithelium, which represent the initial defense barrier, particularly in the context of AR.

It is well known that central to the inflammatory cascade in AR, is an immunoglobulin $\mathrm{E}$ ( $\mathrm{lgE}$ )-dependent response to extrinsic protein antigens orchestrated by eosinophils, T cells, mast cells and basophils, which release several mediators such as chemokines and cytokines ${ }^{(3,4)}$. These mediators are released locally and maintain a persistent inflammation of the nasal mucosa by attracting inflammatory cells and interfering with the mucosal barrier. Recently, studies have indicated that a defective epithelial barrier is a key part of the underlying pathophysiology of diseases such as atopic dermatitis ${ }^{(5)}$, asthma ${ }^{(6)}$, chronic rhinosi- 
nusitis ${ }^{(7)}$, otitis media ${ }^{(8)}$ and $A R^{(9,10)}$.

Although the nasal epithelial cells are the building blocks of the mucosa, their contribution to innate and adaptive immunity only recently started to be understood, particularly in relation to age-dependent maturation ${ }^{(11)}$. The development of the human immune system is a continuous process, while the majority of immune mechanisms are not fully developed from birth ${ }^{(12)}$. In literature, several studies have been conducted in peripheral blood ${ }^{(13)}$, there is very little evidence in epithelial responses at different ages.

This cross-sectional study aimed to evaluate nasal epithelial responses in allergic rhinitis patients of different ages compared to healthy volunteers and characterize the molecular pathways involved.

\section{Materials and methods}

\section{Eligibility criteria and sample collection}

This cross-sectional study enrolled allergic and non-allergic children (0 -18 years) and adults (18-45 years). All subjects were Caucasian. The participants visited P. \& A. Kyriakou Children's hospital and General University Hospital "ATTIKON" for a scheduled clinical evaluation or elective surgery. More specifically, the control group were subjects that were visiting the outpatient department to be evaluated for different conditions or were undergoing elective surgery (orthopaedic, general surgery, urology). The study was approved by the Medical Ethics Review Board of the centers involved (protocol numbers 21826/26-112012 and EB $\Delta$ 528/10-10-13, respectively) and written informed consent was obtained from all participants and/or parents/ legal guardians for children aged less than 18 years. Exclusion criteria specified concomitant chronic illness other than an allergic disease, chronic medication or recent use of medication interfering with an immune response (i.e. $<4$ weeks for systemic corticoid treatment and $<3$ months for other immunomodulatory medicaments or blood product administration), recent $(<4$ weeks) vaccination or acute infection or acute other disease or surgery, smoking and, especially for allergic patients, prior immunotherapy.

A specialist assessed the enrolled participants. Patients with a clinical history compatible with allergic rhinitis and relevant sensitizations, based on the ARIA guidelines ${ }^{(14)}$, were included in the allergic group. Allergic sensitization was assessed by skin prick testing (SPT) to a panel of 8 prevalent local aeroallergens (Dermatophagoides pteronyssinus, Dermatophagoides farinae, cat dander, Alternaria alternata, Cockroach, 5 grasses mix, Olea europaea, Parietaria officinalis) (Alyostal, Stallergenes, France), whereas histamine $(10 \mathrm{mg} / \mathrm{ml})$ and saline were used as a positive and negative control, respectively ${ }^{(15)}$. Wheel diameter of 3 $\mathrm{mm}$ or greater was considered as positive ${ }^{(16)}$. When SPT could not be performed, the sensitization of participants was evaluated by slgE measurement using IMMULITE ${ }^{\circledR} 2000$ immunoassay system (Siemens, Germany) or ImmunoCap allergen-specific IgE blood test (Phadia AB, Uppsala). Values $>0.35 \mathrm{kU} / \mathrm{L}$ were considered positive. Clinical data were recorded in a standardized manner.

Human primary nasal epithelial cell isolation and culture Primary nasal epithelial cells (PNECs) were collected via nasal scrapings [5-10] of the inferior turbinate (both nostrils), utilising a sterile curette (Allgaier Instrumente $\mathrm{GmbH}$, Germany) without local anaesthesia. The PNECs were transferred to collagen (Collagen type I rat tail, Corning USA) coated $25 \mathrm{~cm}^{2}$ flasks and cultured with bronchial epithelial growth medium (BEGM; Lonza; Walkersville, MD, USA), containing $1 \%$ Penicillin/Streptomycin. The growth medium was replaced every $48 \mathrm{~h}$ until a monolayer formed. At passage 2, cells were split onto 24 plates (Corning Costar, USA) and incubated at $37^{\circ} \mathrm{C}$ with $5 \% \mathrm{CO}_{2}$ until $80 \%$ confluence was achieved. Each culture passage occurred every 7 to 10 days.

\section{Protein expression}

Protein expression of multiple cytokines was measured in unstimulated PNECs supernatants when cells reached 70\%-80\% confluency. Twenty-one cytokines were chosen to represent major immune pathway responses. In order to create a network of cytokines that implicate in cell proliferation, immune response discrimination and cell death, the software String 10 (http:// string-db.org/) was used. Enzyme-linked immunosorbent assay (ELISA) was used to detect IL-6 (eBioscience, San Diego, CA, USA), RANTES/CCL5 (R\&D Systems, Oxford, UK) and IFNB1 (R\&D Systems, Oxford, UK) protein concentrations. The absorbance was read and analysed at $450 \mathrm{~nm}$ on a spectrophotometric ELISA plate reader (Sanofi Diagnostics Pasteur, Chaska, USA). Luminex assays for CX3CL1, GCSF, GMCSF, GRO (CXCL1), IL1A, IL1B, IL6, IP10, CCL22, MIP1A MIP1B, PDGFA, TNFA, TRAIL, ENA78, IL20, IL23, IL28A, CCL5, LIF and TSLP, were performed (Millipore Inc, Billerica, MA, USA) according to the manufacturer's instructions. Briefly, supernatants were incubated with antibody-coated magnetic beads, followed by the addition of a biotinylated detection antibody. Proteins were detected by incubation with phycoerythrin-labelled streptavidin, and the resultant bead immuno-complexes were read on XPONENT ${ }^{\circledR}$ Software (Luminex, TX, USA). Median fluorescence intensity (MFI) was collected and used for calculating protein concentration.

\section{Statistical analysis}

Initially, all continuous variables (demographic and cytokines) were tested for normality with the Shapiro-Wilk test, and they were all found to be non-normal. Based on that, non-parametric tests were performed on them, and their descriptives were provided in the form of "median (25th-75th percentile)". In order to perform cytokine comparisons among two groups (control 
Table 1. The demographic characteristics of both healthy subjects, and subjects with allergic rhinitis. Regarding qualitative characteristics, a count (\% relative frequency) is provided per variable level, while comparison among the two groups is performed with Pearson's chi-squared test of independence. Regarding quantitative variables, descriptives are given in the form of median (25th - 75th percentile), and the $p$-value is extracted with Wilcoxon's rank-sum test.

\begin{tabular}{|c|c|c|c|}
\hline Variable & $\begin{array}{l}\text { Control group descriptives } \\
\qquad(n=33)\end{array}$ & $\begin{array}{l}\text { Allergic rhinitis descriptives } \\
\qquad(n=14)\end{array}$ & p-value \\
\hline Age, years, median (range) & $7.92(2.75-13.83)$ & $14.96(8.1-22.95)$ & 0.045 \\
\hline $\begin{array}{r}\text { Gender } \\
\text { male } \\
\text { female }\end{array}$ & $\begin{array}{l}13(39.39) \\
20(60.61)\end{array}$ & $\begin{array}{l}10(71.43) \\
4(28.57)\end{array}$ & 0.091 \\
\hline BMI & $18.1(15.7-22.7)$ & $19.75(16-25.17)$ & 0.5 \\
\hline 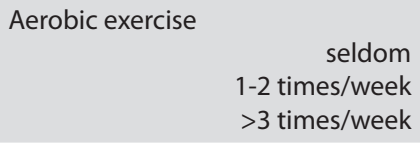 & $\begin{array}{c}7(29.17) \\
10(41.67) \\
7(29.17)\end{array}$ & $\begin{array}{l}2(14.29) \\
5(35.71) \\
7(50)\end{array}$ & 0.378 \\
\hline $\begin{array}{r}<1 \text { hour/day } \\
1-3 \text { hours/day } \\
3-5 \text { hours/day } \\
>5 \text { hours/day }\end{array}$ & $\begin{array}{l}9(33.33) \\
9(33.33) \\
8(29.63) \\
1(3.7)\end{array}$ & $\begin{array}{l}4(28.57) \\
9(64.29) \\
0(0) \\
1(7.14)\end{array}$ & 0.092 \\
\hline Smoking & $\begin{array}{c}33(100) \\
0(0)\end{array}$ & $\begin{aligned} 13 & (92.86) \\
1 & (7.14)\end{aligned}$ & 0.655 \\
\hline $\begin{array}{l}\text { Smokers in house } \\
\text { (One or two or more) } \\
\text { Parental smoking cigarettes per } \\
\text { day (father or mother or both) }\end{array}$ & $\begin{array}{c}48.48 \% \\
15(9.5-20)\end{array}$ & $\begin{array}{c}42,85 \% \\
20(20-25)\end{array}$ & $\begin{array}{l}0.826 \\
0.057\end{array}$ \\
\hline $\begin{array}{l}\text { Traffic burden } \\
\text { (Living in medium- and high-traffic } \\
\text { street) }\end{array}$ & $78,78 \%$ & 71,42 & 0.421 \\
\hline Pets in house & $\begin{array}{l}21(65.62) \\
11(34.38)\end{array}$ & $\begin{array}{l}10(71.43) \\
4(28.57)\end{array}$ & 0.964 \\
\hline Humidity in house & $\begin{array}{l}19(59.38) \\
13(40.62)\end{array}$ & $\begin{array}{l}11(78.57) \\
3(21.43)\end{array}$ & 0.357 \\
\hline Nutritional supplements & $\begin{array}{c}31(96.88) \\
1(3.12)\end{array}$ & $\begin{array}{c}13(92.86) \\
1(7.14)\end{array}$ & 1 \\
\hline Daycare school attendance & $\begin{array}{c}8(32) \\
17(68)\end{array}$ & $\begin{array}{c}0(0) \\
10(100)\end{array}$ & 0.112 \\
\hline Breastfeeding & $\begin{array}{c}5(17.86) \\
23(82.14)\end{array}$ & $\begin{array}{c}2(15.38) \\
11(84.62)\end{array}$ & 1 \\
\hline
\end{tabular}

vs AR), Wilcoxon's rank-sum test (equivalent to Mann-Whitney test) was applied. Pearson's chi-squared test of independence was used to recognize any dependency between categorical variables. Linear regression analysis was performed using all the patients (both control and AR groups), associating jointly the age and the AR status with the levels of the cytokines' expression in order to evaluate their combined effect.

All statistical tests were considered as two-sided and statistical significance was taken at the level of $5 \%$. Analysis was performed with the R statistical software and the RStudio interface, both open-source products.
Bioinformatic pathway analysis

We performed pathway analysis using the Functional Annotation Tool DAVID Bioinformatics Resources 6.8, NIAID/NIH, considering proteins statistically significantly different between AR patients and controls.

\section{Results}

Subjects' characteristics and PNECs cultures

A total of 59 subjects were included ( 39 normal subjects and 20 subjects with allergic rhinitis - (AR)). The PNECs from 47 subjects $(79,66 \%)$ were successfully cultured, and their unstimulated 
Table 2. Comparing the protein expression levels between control and AR PNECs; the comparisons have been performed with Wilcoxon's rank-sum test, while descriptive statistics are given in the form of median (25th - 75th percentile).

\begin{tabular}{|c|c|c|c|}
\hline variable & $\begin{array}{l}\text { Control group median } \\
\text { (25-75 percentile) }\end{array}$ & $\begin{array}{l}\text { AR group median } \\
\text { ( } 25-75 \text { percentile) }\end{array}$ & p-value \\
\hline GMCSF & $426.6(191.77$ - 998.03) & $191.92(84.9$ - 292.65) & 0.003 \\
\hline MIP1B & $10.83(9.08$ - 14.96) & $8.58(5.23-9.82)$ & 0.012 \\
\hline TNFA & $34.42(15.77-55.81)$ & $14.64(12.5-24.65)$ & 0.016 \\
\hline CCL5 & $20.72(9.59-38.76)$ & $10.05(6.29-15.98)$ & 0.033 \\
\hline IL28A & $9.31(6.87-12.46)$ & $6.59(5.16-7.61)$ & 0.037 \\
\hline MIP1A & $5.03(4.18-7.35)$ & $4.25(3.49-4.69)$ & 0.042 \\
\hline IL6 & $770.91(457.16-1011.06)$ & $562.24(479.87-682.57)$ & 0.131 \\
\hline IP10 & $221.22(119.55-338.02)$ & 167.4 (88.71 - 239.69) & 0.15 \\
\hline LIF & $13.6(10.53-17.01)$ & $11.37(8.68$ - 14.92) & 0.152 \\
\hline TRAIL & $1.88(1.31-2.39)$ & $2.25(1.75-2.76)$ & 0.173 \\
\hline IL1B & $62.64(25.5-118.5)$ & $39.73(10.63-90.55)$ & 0.247 \\
\hline CX3CL1 & 33.77 (30.17 - 41.84) & $27.87(25.37-43.7)$ & 0.288 \\
\hline IL23 & $11.43(0-37.45)$ & $30.49(4.62-45.1)$ & 0.296 \\
\hline IL20 & $64.58(53.91-91.52)$ & $71.07(60.31-105.47)$ & 0.403 \\
\hline PDGFA & $655.52(308.5$ - 1467.13) & $1080.2(264.07-1723.9)$ & 0.516 \\
\hline IL1A & $187.27(125.78-308.15)$ & $156.15(108.41-275.11)$ & 0.531 \\
\hline GCSF & $2294.2(1271.79-8370.74)$ & $5745.66(812.2$ - 9915.84) & 0.659 \\
\hline TSLP & $20.92(14.98-27.61)$ & $20.33(7.99-27.31)$ & 0.676 \\
\hline ENA.78 & $239.19(148.96$ - 1019.99) & $369.85(170.58$ - 724.28) & 0.729 \\
\hline MDC (CCL22) & $8.75(1.39-14.2)$ & $9.94(5.3-12.95)$ & 0.764 \\
\hline GRO & $7297.39(5021.25$ - 8174.08) & $6971.37(6330.98-7679.25)$ & 0.897 \\
\hline
\end{tabular}

supernatants were analyzed for 21 cytokines and chemokines. The percentage of AR PNECs successfully cultured up to the third passage was $70 \%$ (14/20), slightly lower than controls (33/39-84\%).

The demographic characteristics of participants are shown in Table 1. The general characteristics of the groups were similar with the exception of the age in AR patients which was higher than in controls (age AR patients Vs controls: 14.96 years old Vs 7.92 years old $p$-value 0,045 ).

Also, a marginal difference ( $p=0.057)$ was detected between AR patients and control group according to parental smoking cigarettes per day: parents of AR patients were smoking more $(>20)$ cigarettes per day than parents of controls $(<20)$.

\section{Differentially expressed proteins between PNECs from AR} patients and control individuals

Comparing the protein expression levels between control and AR PNECs, several mediators appeared to be decreased in subjects with allergic rhinitis (Table 2). Six of them (GMCSF, MIP1A, MIP1B, IL28A, TNFA, CCL5) (Figure 1) were statistically significantly lower in the AR subjects than the control group. Further, we analyzed the data according to age, comparing either two groups (adults vs children $<18)$ ) or three groups: children $(0-$ 12yrs), adolescence (>12 - 18yrs) and adults (Table S1). As shown in Table S1, three of the cytokines (GMCSF, IL28A, TNFA) were significantly lower in children with allergic rhinitis than controls $(p<0.05)$. Only one chemokine was differentially expressed in adults, MIP1B. MIP1B is lower in the adult AR group than adults' controls $(p<0.05)$.

When comparing children (<12yrs) and adolescents ( $>12-18 \mathrm{yrs})$, the most prominent differences in protein expression were detected in the age group of $<12$ years old, as seen in Table S1. Three cytokines (GMCSF, MIP1A, IL28A) are statistically significantly lower expressed only in the group of $A R<12$ years old compared to the control group. There were no differences in adolescences. None of the mediators studied was expressed statistically significantly higher in AR patients compared to controls.

Furthermore, taking into account the age difference between control and AR subjects ( $p=0.045$; Table 1 ), we performed linear regression analysis using all the patients (both control and $A R$ groups), associating jointly the age and the AR status with the levels of the cytokines' expression that were significantly differentiated among groups in order to evaluate their combined 


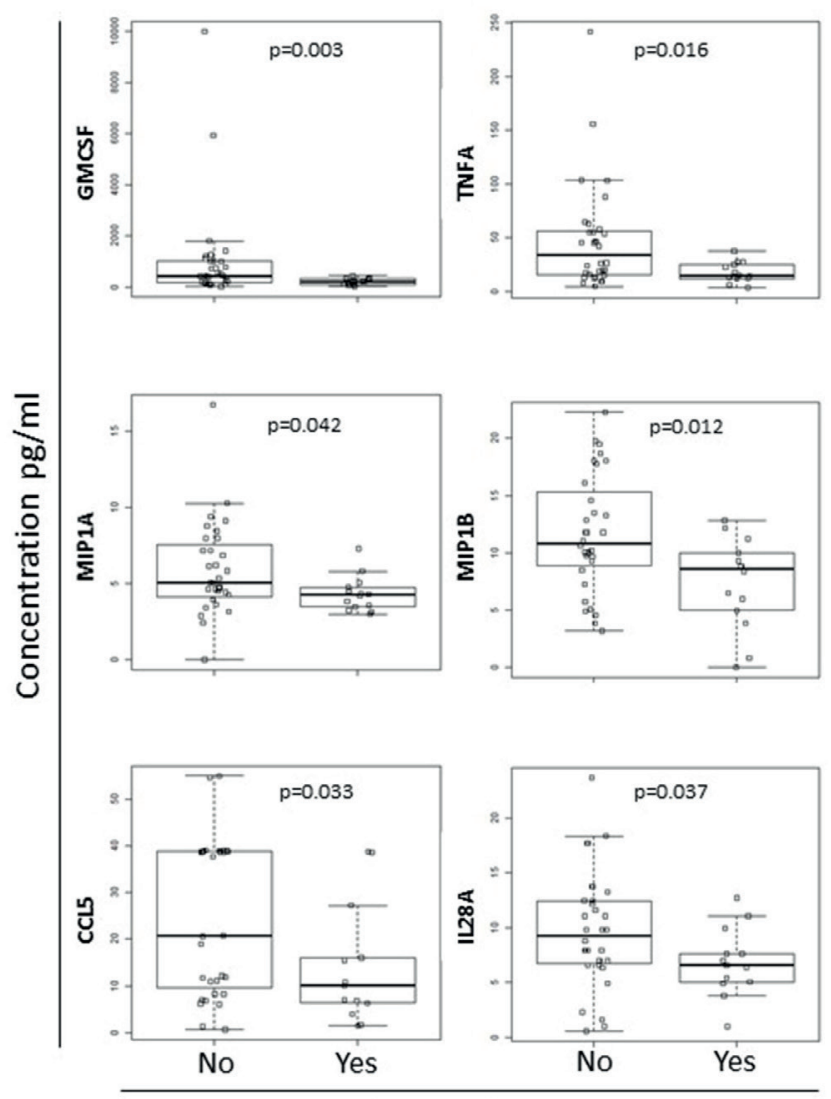

Allergic Rhinitis

Figure 1. A set of 6 side-by-side boxplots combined with strip-chart visualizations that illustrate the statistically significantly dissimilar protein expression levels of control and AR subjects, accompanied with the $\mathrm{p}$-values of the comparisons.

effect. The results showed that age was found to be a significant predictor only to IL28A ( $p=0.04)$, independently of disease status (control or AR), (GMCSF $p=0.26$, MIP1A $p=0.60$, MIP1B $p=0.95$, TNFA $p=0.09, C C L 5=0.27$ ). We also performed linear regression analysis separately for controls and AR using IL28A levels and age and we did not find any difference. It seems that the age is the main factor of IL28A differentiation, and this may influence the results in the group of children. In addition, removing from the analysis the subjects under 3 years old, the statistical difference of IL28A between AR and controls was $p=0.07$, still remaining a trend of difference (Figure S1).

\section{Pathway analysis in differentially expressed proteins} between PNECs from AR patients and healthy individuals We performed pathway analysis using the Functional Annotation Tool DAVID Bioinformatics Resources 6.8 in statistically significantly different proteins between allergic rhinitis patients and controls. The pathways identified include key inflammatory and innate immune response pathways. Cytokine-cytokine receptor interaction, TNF signaling pathway, NOD-like receptor signaling pathway, Influenza A, Chemokine signaling pathway, and Toll-like receptor signaling pathway were found significantly downregulated in allergic rhinitis $(p<0.05)$ (Table 3$)$.

\section{Discussion}

In the last decades, the airway epithelium, which is in the interface between the external and internal environment, has gained much attention as its dysfunction may cause the pathogenesis of many diseases. More specifically, multiple studies highlight the significant role of the airway epithelium for an appropriate immune response through the production of a cascade of cytokines and chemokines that activate inflammatory cells to neutralize inhaled allergens, infectious agents, or pollutants. However, the studies in patients with allergic rhinitis focusing on the nasal epithelium and its role in the innate immune response are limited.

This study was designed to explore the baseline cytokine profile of nasal epithelium of healthy subjects and subjects with allergic rhinitis of different ages. Our key finding is that the spontaneous secretion of specific cytokines (MIP1A, MIP1B, IL28A, CCL5, TNFA and GMCSF) is lower in patients with allergic rhinitis than in healthy controls. These cytokines are involved in the main pathways involving innate immune responses, specifically in viral infections (NOD-like receptor signaling pathway, Influenza A, Toll-like receptor signaling pathway). In the present study, it is the first time, we map these pathways in health and show interesting differences in children with allergic rhinitis. These differences were more prominent in children, while MIP1B was lower in adults. Our results suggest that there is a dysfunction of mucosal immune pathways in patients with allergic rhinitis. Based on the one airway -one disease theory, nasal epithelial cells have been used as surrogates to bronchial epithelial cells for research purposes investigating the mechanism of wheezing and asthma. In a recent study, nasal epithelial cells from neonates were collected and cultured. The parents of the neonates were contacted with a questionnaire when their children turned 4 years old. It is reported that at birth, supernatants from cultured cells from the children with recent wheeze had reduced release of IL-8 IL-6, GMCSF, and ICAM-1 after treatment with culture medium alone, with TNFa/IL-1 $\beta$ and LPS compared to children without wheeze ${ }^{(17)}$. Further investigations ${ }^{(18)}$ in older children (up to 16 years old) demonstrated that, at baseline, nasal epithelial cells from children with a history of wheeze produced significantly less IL-8, IL-6, MCP-1, and G-CSF than healthy controls. After stimulation with IL-1 $\beta$ and TNFa, cells from children with current wheeze produced significantly less IL8, IL-6, and MCP-1 proteins than control children. In both these studies, it is suggested that abnormal epithelial cytokine release may play an essential role in the pathophysiology of wheeze, and as evident in our study, this may also present in patients with allergic rhinitis. 
Table 3. Pathway analysis of differential expressed genes between allergic rhinitis and healthy PNECs.

\begin{tabular}{llllll}
\hline & & & & & \\
\hline KEGG_PATHWAY & pon05323:Rheumatoid arthritis & 6 & 1.587302 & 0.00 & CSF2, CCL5, CCL3, CCL2, TNF \\
\hline KEGG_PATHWAY & pon04060:Cytokine-cytokine receptor interaction & 6 & 1.587302 & 0.00 & CSF2, CCL5, CCL3, CCL2, TNF \\
\hline KEGG_PATHWAY & pon05142:Chagas disease & 5 & 1.322751 & 0.00 & CCL5, CCL3, CCL2, TNF \\
\hline KEGG_PATHWAY & pon04668:TNF signaling pathway & 5 & 1.322751 & 0.00 & CSF2, CCL5, CCL2, TNF \\
\hline KEGG_PATHWAY & pon04621:NOD-like receptor signaling pathway & 4 & 1.058201 & 0.00 & CCL5, CCL2, TNF \\
\hline KEGG_PATHWAY & pon05164:Influenza A & 4 & 1.058201 & 0.00 & CCL5, CCL2, TNF \\
\hline KEGG_PATHWAY & pon04062:Chemokine signaling pathway & 4 & 1.058201 & 0.00 & CCL5, CCL3, CCL2 \\
\hline KEGG_PATHWAY & pon05168:Herpes simplex infection & 4 & 1.058201 & 0.00 & CCL5, CCL2, TNF \\
\hline KEGG_PATHWAY & pon05144:Malaria & 3 & 0.793651 & 0.00 & CCL2, TNF \\
\hline KEGG_PATHWAY & pon04620:Toll-like receptor signaling pathway & 3 & 0.793651 & 0.00 & CCL5, CCL3, TNF \\
\hline KEGG_PATHWAY & pon04664:Fc epsilon RI signaling pathway & 2 & 0.529101 & 0.05 & CSF2, TNF \\
\hline KEGG_PATHWAY & pon04640:Hematopoietic cell lineage & 2 & 0.529101 & 0.06 & CSF2, TNF \\
\hline KEGG_PATHWAY & pon05132:Salmonella infection & 2 & 0.529101 & 0.06 & CSF2, CCL3 \\
\hline KEGG_PATHWAY & pon04660:T cell receptor signaling pathway & 2 & 0.529101 & 0.07 & CSF2, TNF \\
\hline KEGG_PATHWAY & pon05146:Amoebiasis & 2 & 0.529101 & 0.08 & CSF2, TNF \\
\hline KEGG_PATHWAY & pon04650:Natural killer cell mediated cytotoxicity & 2 & 0.529101 & 0.08 & CSF2, TNF \\
\hline & & & & \\
\hline
\end{tabular}

The question of whether reduced cytokine release from PNECs in subjects with allergic rhinitis is a result of environmental stress or inflammation or genetic defect is still unclear ${ }^{(19)}$. Nevertheless, any reduction in mediator production may result in a suboptimal response of the epithelium and potential dysfunction of the epithelial barrier. It is well accepted that cytokines not only activate inflammatory cells but also regulate the epithelial barrier in allergic disease ${ }^{(20,21)}$. Cytokines, such as IL13, may alter tight junctions (TJs) of the mucosa, which play a key role in the mucosal barrier ${ }^{(22,23)}$. Our findings of reduced cytokine release from the nasal epithelial cell in the allergic population may suggest a distraction of the whole cytokine cascade and/or other sources of cytokines, such as neutrophils and macrophages, may replace the epithelial cell gap ${ }^{(18)}$.

It is well accepted that the development of the human immune system is a continuous process. On the other hand, it has been suggested that the reduced cytokine production from nasal epithelial cells of subjects with allergic rhinitis compared to normal subjects may represent in vitro differences of the cultured cells. Cytokine release differences may represent an in vitro different state of differentiation of the epithelial cells from the allergic population ${ }^{(18)}$. This may also explain the difference between the two groups in the success rate of the epithelial cells cultures in our study. In addition, these differences may reflect an in vivo altered differentiation pattern or may be reflect a delayed maturation process. Based on the above, our findings may support the theory that the allergic nasal epithelium cannot undergo standard mechanisms of repair and differentiation.

Finally, our results suggest that in children, allergy may compromise the mechanical and immunological defense function of the nasal mucosa against viruses ${ }^{(24)}$. All the inflammatory mediators differentially expressed in the allergic population of our study are essential to the host response infection. The cytokines MIP1A, MIP1B and CCL5/RANTES have strong chemotactic effects on many inflammatory cells such as monocytes, $T$ lymphocytes and neutrophils ${ }^{(25,26)}$. The GMCSF, released by the airway epithelium, along with IL-4 and tumor necrosis factor- $a$ (TNF- $a$ ) cause DC maturation which then these cells are actively involved in the development and maintenance of an inflammatory response ${ }^{(27)}$. TNF- $a$ is an inflammatory cytokine produced by macrophages/monocytes during acute inflammation and is responsible for a diverse range of signaling events within cells, leading to necrosis or apoptosis. It is also crucial for resistance to infection and cancers ${ }^{(28)}$. IL28A is essential for maintaining the integrity and homeostasis of epithelial tissue layers. It promotes innate immune responses from tissue epithelia to limit the damage caused by viral and bacterial infections ${ }^{(29)}$. IL28A was highlighted as the most age-related cytokine, and this may influence the results in the group of children. The fact that AR is an allergic disease that is present in older ages ( $>3$ years), may influence the significancy of the results. Although, all the literature about the interferon deficiency in allergy upon virus stimulation supports that is due to allergic status without taking into consideration the age. In agreement with our results, Degir- 
menci et al., reported IFN- $\gamma$ deficiency in AR adults compared to controls ${ }^{(30)}$.

Our study has several limitations. The nature of our data is crosssectional, and as a result, inter-individual variability could play a significant role in the observed differences in the cytokine profiles. Every effort was made to eliminate factors that affect cytokines release by applying strict inclusion criteria. The number of subjects in each group is low due to the significant cost and effort required for primary cell cultures. However, this is the first study of age-stratified comparison of mediators of nasal epithelial cells in allergic rhinitis and control subjects to the best of our knowledge.

\section{Conclusion}

Based on the above, we suggest that patients with allergic rhinitis, especially the young ones, have an altered epithelial cytokine profile, possibly related to abnormal immune maturation, supporting disease predisposition. These observations highlight the need to investigate further inflammatory pathways in the pathogenesis of the allergic response, which may lead to more effective early intervention in allergic rhinitis.

\section{Authorship contribution}

SS did the sampling, cell culture and wrote the paper. PNG conceived of the presented idea. LI performed statistical analysis. GA, MP and X did the clinical evaluation of the cohort. AE verified the analytical methods. PE supervised the findings of this work. LE did the contributed to writing and editing the paper. TS, analysed the cytokines, supervised the project and created the concept of this publication. All authors discussed the results and contributed to the final manuscript.

\section{Acknowledgments}

None.

\section{Funding}

The research project was supported by the Hellenic Foundation for Research and Innovation (H.F.R.I.) under the "1st Call for H.F.R.I. Research Projects to support Faculty Members \& Researchers and the Procurement of High-and the procurement of high-cost research equipment grant" (Project Number: HFRI-

FM17- 3063).

\section{Ethics approval and consent to participate}

Not applicable.

\section{Consent for publication}

Not applicable.

\author{
Availability of data and materials \\ Not applicable.
}

\section{Conflict of interest}

There is no conflict of interest.

\section{References}

1. Prokopakis E, Vardouniotis A, Bachert C, et al. Rhinology Future Debates 2018 a EUFOREA Report. Rhinology. 2020 58(4):384-393.

2. Bousquet J, Hellings PW, loana Agache I, et al. Allergic Rhinitis and its Impact on Asthma (ARIA) Phase 4 (2018): Change management in allergic rhinitis and asthma multimorbidity using mobile technology. J Allergy Clin Immunol. 2019 Mar;143(3):864 879

3. Steelant B, Seys SF, Boeckxstaens G, Akdis CA, Ceuppens JL, Hellings PW. Restoring airway epithelial barrier dysfunction: a new therapeutic challenge in allergic airway disease. Rhinology. 2016 Sep;54(3):195-205

4. Bartlett JA, Fischer AJ, McCray Jr PB. Innate Immune Functions of the Airway Epithelium. Contrib Microbiol. 2008; vol 15, pp 147-163.

5. Benedetto AD, Rafaels NM, McGirt LY, et al. Tight Junction Defects in Atopic Dermatitis. J Allergy Clin Immunol. 2011 Mar; 127(3): 773-786.

6. Xiao G, Puddicombe SM, Field S, et al. Defective epithelial barrier function in asthma. J Allergy Clin Immunol. 2011 Sep;128(3):549-56.
7. Soyka MB, Wawrzyniak P, Eiwegger T, et al. Defective epithelial barrier in chronic rhinosinusitis: the regulation of tight junctions by IFN- $\gamma$ and IL-4. J Allergy Clin Immunol. 2012 Nov;130(5):1087-1096.

8. Chantzi FM, Kafetzis DA, Bairamis T, et al. IgE sensitization, respiratory allergy symptoms, and heritability independently increase the risk of otitis media with effusion. Allergy. 2006 Mar;61(3):332-6

9. Steelant B, Farré R, Wawrzyniak $P$, et al. Impaired barrier function in patients with house dust mite-induced allergic rhinitis is accompanied by decreased occludin and zonula occludens-1 expression. J Allergy Clin Immunol. 2016 Apr;137(4):1043-1053.

10. Lee HJ, Kim B, Im NR, et al. Decreased expression of E-cadherin and ZO-1 in the nasal mucosa of patients with allergic rhinitis: Altered regulation of E-cadherin by $\mathrm{LL}-4$, IL-5, and TNF-alpha. Am J Rhinol Allergy. 2016 May; 30(3):173-8

11. Xatzipsalti M, Psarros F, Konstantinou G, et al. Modulation of the epithelial inflammatory response to rhinovirus in an atopic environment. Clin Exp Allergy. 2008 Mar;38(3):466-72

12. Georgountzou A, Papadopoulos NG. Postnatal Innate Immune Development:
From Birth to Adulthood. Front Immunol. 2017 Aug; 11:8-957.

13. Georgountzou A, Kokkinou D, Taka S, et al. Differential maturation trajectories of innate antiviral immunity in health and atopy. Pediatr Allergy Immunol. 2021 Jul; 20.

14. Bousquet PJ, Khaltaev N, Cruz AA, et al. Allergic Rhinitis and its Impact on Asthma (ARIA) 2008 update (in collaboration with the World Health Organization, GA(2)LEN and AllerGen). Allergy. 2008 Apr;63 Suppl 86:8-160.

15. Haahtela T, Burbach GJ, Bachert C, et al Clinical relevance is associated with allergen-specific wheal size in skin prick testing. Clin Exp Allergy. 2014 Mar;44(3):407-16

16. Konstantinou GN, Bousquet PJ, Zuberbier T, Papadopoulos NG. The longest wheal diameter is the optimal measurement for the evaluation of skin prick tests. Int Arch Allergy Immunol. 2010; 151(4):343-5.

17. Turner S, Miller D, Walsh GM, et al. Proinflammatory mediator responses from neonatal airway epithelial cells and early childhood wheeze. Pediatric Pulmonology. 2017; Vols. 1-7.

18. McDougall CM, Helms PJ, Walsh GM. Airway epithelial cytokine responses in childhood wheeze are independent of atopic status. 
Respiratory Medicine. 2015;109: 689-700.

19. Hellings PW, Steelant B. Epithelial barriers in allergy and asthma. J Allergy Clin Immunol. 2020 Jun;145(6):1499-1509.

20. Steelant B, Seys SF, Van Gerven L, et al. Histamine and $T$ helper cytokine-driven epithelial barrier dysfunction in allergic rhinitis. J Allergy Clin Immunol. 2018 Mar;141(3):951-963.

21. Heijink IH, Nawijn MC, Hackett TL. Airway Epithelial Barrier Function Regulates the Pathogenesis of Allergic Asthma. Clin Exp Allergy.2014; 44:620-30.

22. London NR Jr, Tharakan A, Ramanathan $M$ Jr. The Role of Innate Immunity and Aeroallergens in Chronic Rhinosinusitis. Adv Otorhinolaryngol. 2016; 79:69-77.

23. Nur Husna SM, Tina Tan HT, Shukri N, Mohd Ashari NS, Wong KK. Nasal Epithelial Barrier Integrity and Tight Junctions Disruption in Allergic Rhinitis: Overview and Pathogenic Insights. Front Immunol. 2021; 12: 663626

24. De Corso E, Lucidi D, Cantone E, et al. Clinical Evidence and Biomarkers Linking
Allergy and Acute or Chronic Rhinosinusitis in Children: A Systematic Review. Curr Allergy Asthma Rep. 2020 Sep;20(11):68.

25. Menten P, Wuyts A, Van Damme J. Macrophage inflammatory protein-1. Cytokine Growth Factor Rev. 2002 Dec;13(6):455-81.

26. Marques RE, Guabiraba R, Russo RC Teixeira MM. Targeting CCL5 in inflammations. Expert Opin Ther Targets. 2013 Dec;17(12):1439-60

27. Tyurin YA, Lissovskaya SA, Fassahov RS, et al. Cytokine Profile of Patients with Allergic Rhinitis Caused by Pollen, Mite, and Microbial Allergen Sensitization. J Imm Res. 2017; 22017:3054217.

28. Idriss HT, Naismith JH. TNF alpha and the TNF receptor superfamily: structure-function relationship(s). Microsc Res Tech. 2000 Aug 1;50(3):184-95.

29. Ouyang W, Rutz S, Crellin NK, Valdez PA, Hymowitz SG. Regulation and functions of the IL-10 family of cytokines in inflammation and disease. Annu Rev Immunol. 2011;
29:71-109.

30. Degirmenci PB, Aksun S, Altin Z et al. Allergic Rhinitis and Its Relationship with IL-10, IL-17, TGF- $\beta$, IFN- $\gamma$, IL 22, and IL-35. Disease Markers. 2018; 9131432.

\section{S. Stamataki}

ENT dept.

Childrens' Hospital "Agia Sofia"

Athens

Greece

Tel: +302132007310

E-mail: sofiastamataki@gmail.com 


\section{SUPPLEMENTARY DATA}

Table S1. Comparing the protein expression levels between control and AR PNECs in different age groups (0-12, $>12-18,<18$ and $>18$ years old); the comparisons have been performed with Wilcoxon's rank-sum test, while descriptive statistics are given in the form of median (25th - 75th percentile).

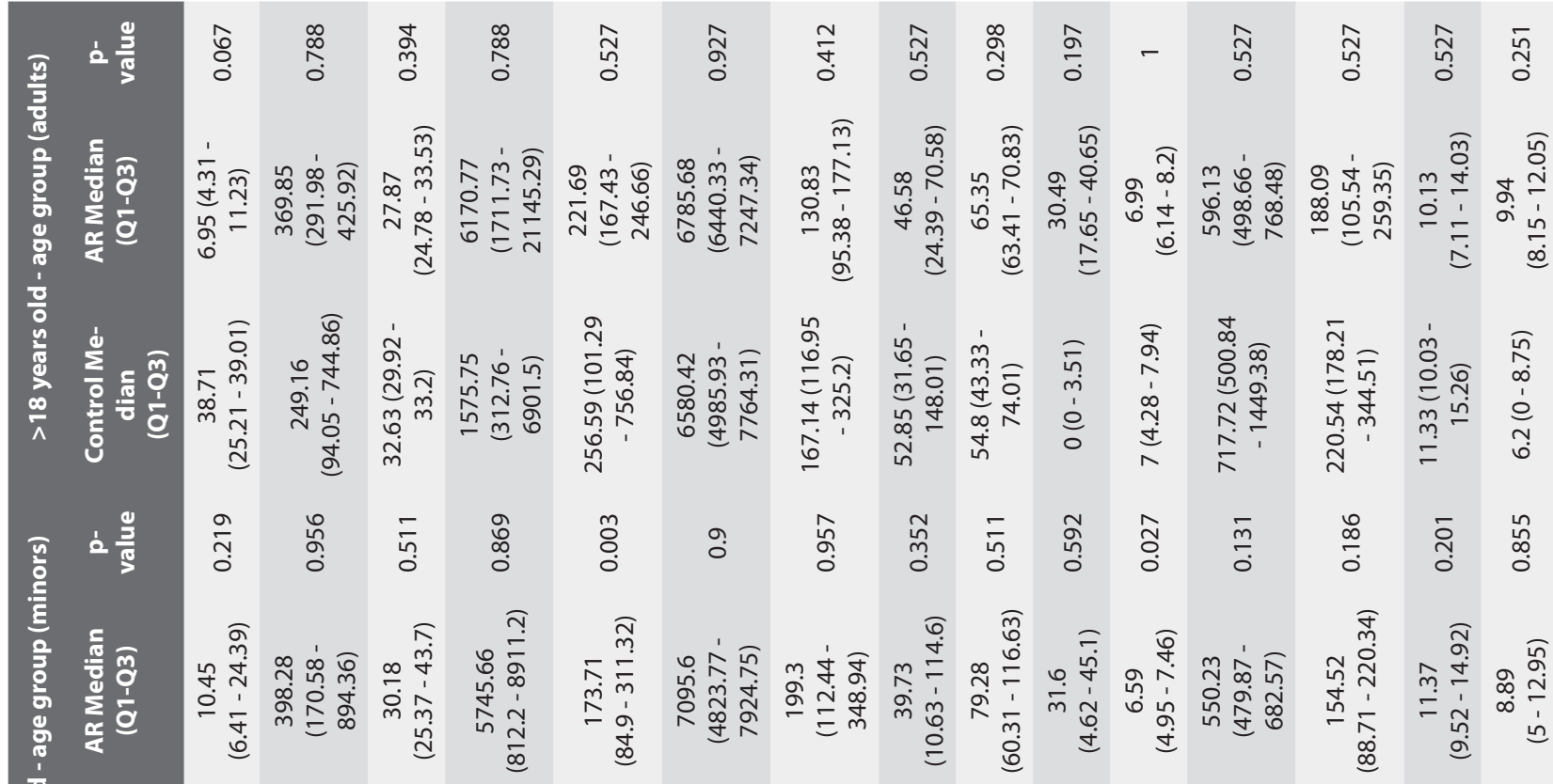

定

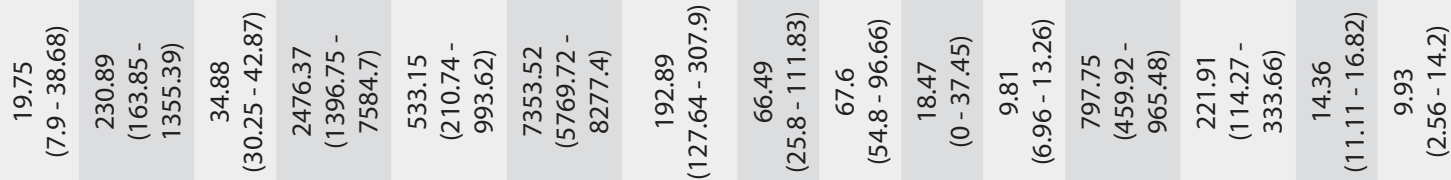

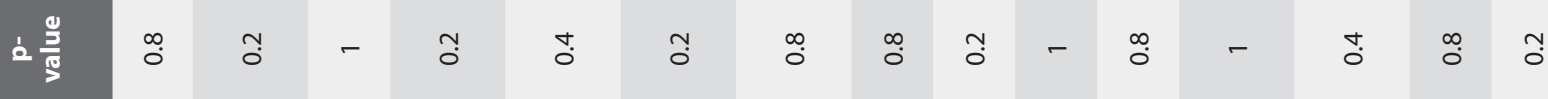

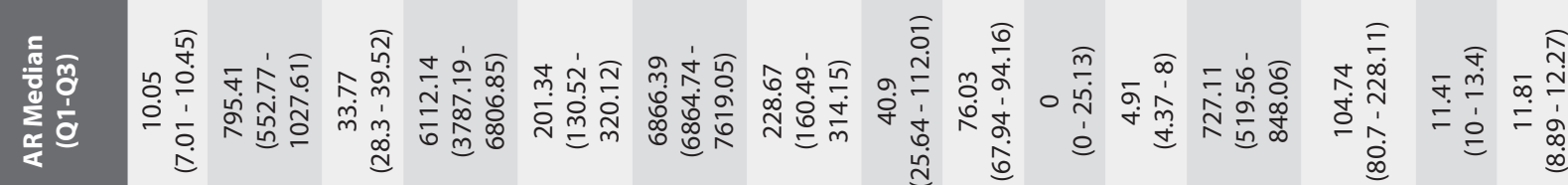

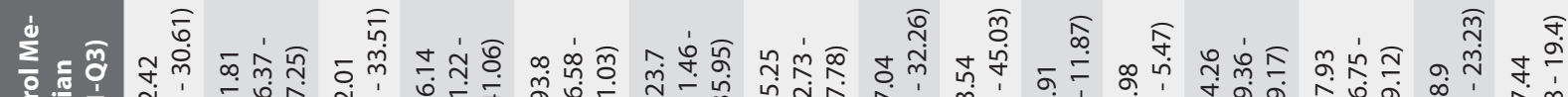

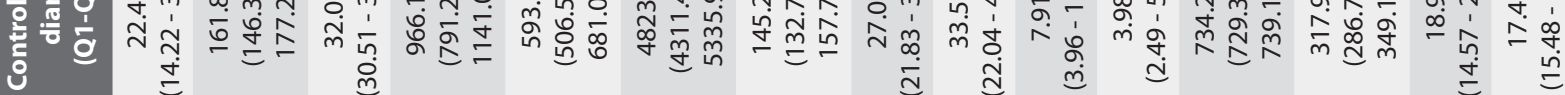

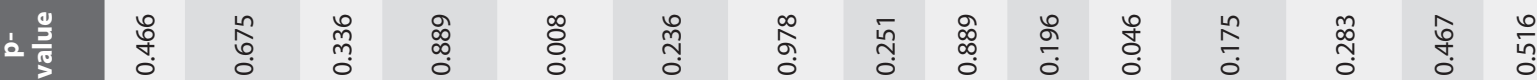

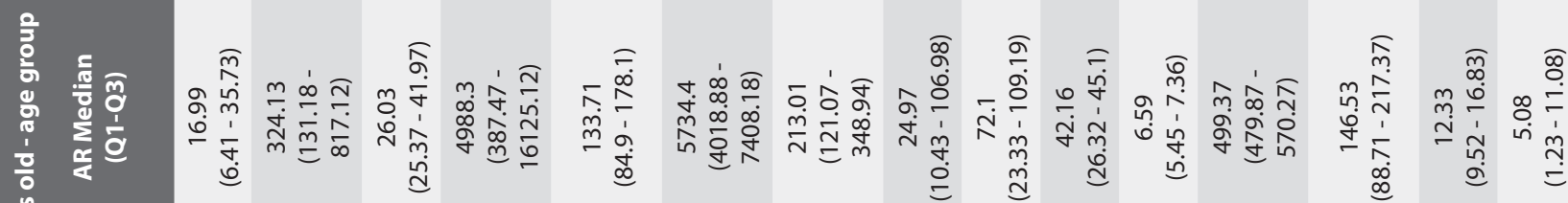

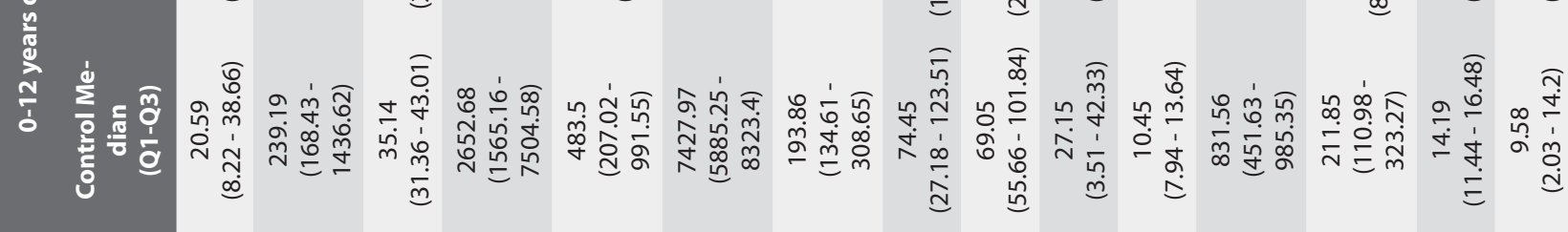
氙竞 


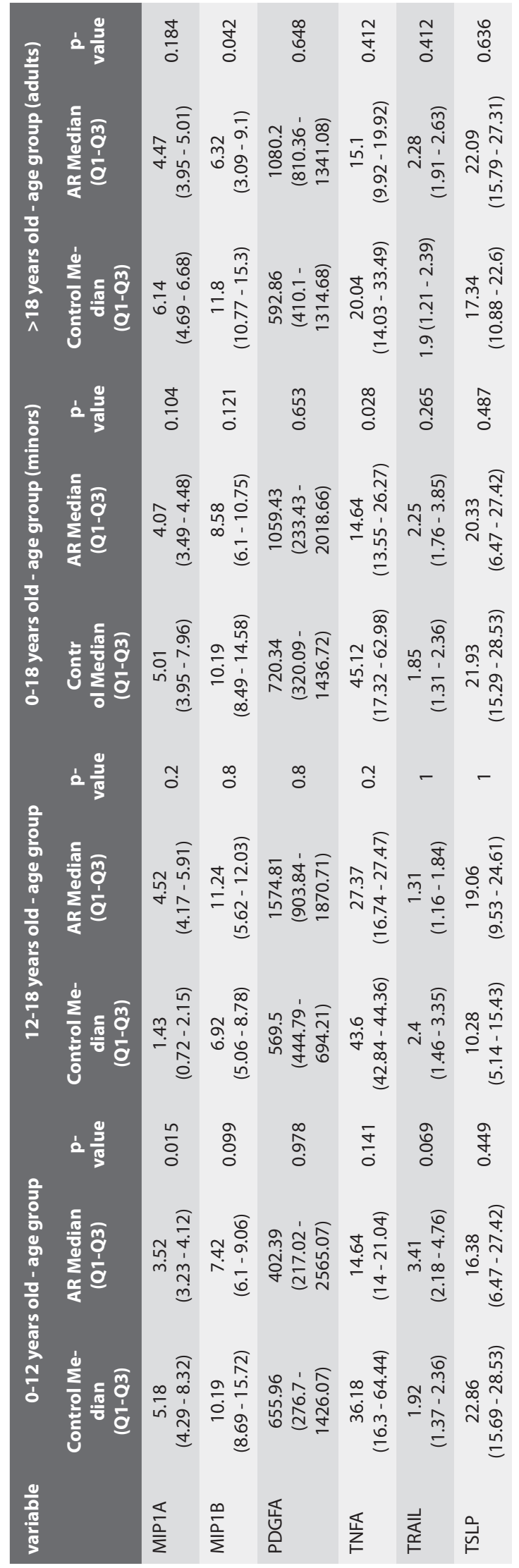

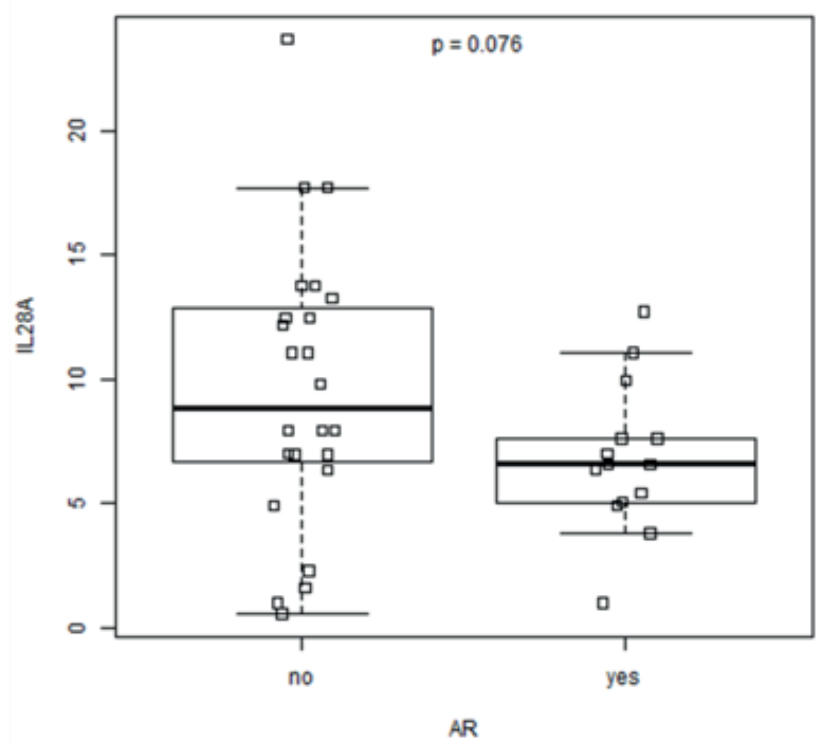

Figure S1: Boxplot combined with strip-chart visualizations that illustrate the IL28A protein expression levels of control ( $>3$ years old) and AR subjects, accompanied with the $p$-value of the comparison. 